\title{
Reconstruction of Wideband Radar Signal Based on Compressed Sensing
}

\author{
Li Wenjuan $^{1}$ and Yang Haolan ${ }^{2}$ \\ ${ }^{1}$ College of Mobile Telecommunications Chongqing University of Posts and \\ Telecom, Hechuan, Chongqing, China \\ ${ }^{2}$ College of Optoelectronic Engineering Chongqing University of Posts and \\ Telecom, Nanan, Chongqing, China
}

\begin{abstract}
The matched filter is often used for the radar receiving terminal, but such application in wideband radar may cause complex system and high cost. The adoption of compressed sensing can not only remove the matched filter and the high-speed analogdigital converter, but also realize high-resolution radar imaging. In consideration of the sparseness of the radar target scene, a compressed sensing method used for chaotic radar is proposed in this article, wherein the chaotic frequency-modulated signal is generated by Bernoulli mapping and the randomness of such signal can be directly used for constructing the observation matrix. Meanwhile, the under-sampled echo signal can be used for radar scene reconstruction through the optimization technology. According to the simulation of stationary and non-stationary target radar scenes, compared with the matched filter processing technology, the proposed method has simple processing procedure and optimal performance, and the feasibility of this method is also verified by the simulation result.
\end{abstract}

Keywords: Chaotic Radar; Wideband Radar; Compressed Sensing; Matched Filter

\section{Introduction}

High-resolution radar imaging technology is significant for radar target identification and feature extraction. According to radar imaging principle [1, 2], the distance resolution of the target depends on the bandwidth of the transmitted signal while the azimuth resolution relies on phase-coherent accumulation time. Constrained by Nyquist sampling theorem, the traditional wideband radar imaging system encounters such problems as high sampling rate, large data volume and echo data limitation.

CS (Compressed Sensing) [3-7] theory provides a new approach for wideband radar imaging. This theory shows: for a sparse signal, the original signal can be accurately or approximately reconstructed through a few times of observations, namely through signal projection values. The result of the research on the electromagnetic scattering characteristics of the radar target shows [8] that the pulse response of the radar target in the high-frequency area can be described by a few important scattering centers, and the number of the important scattering centers obtained by the wideband radar is much less than the number of the samples needed for identifying these scattering centers. Obviously, the wideband radar target characteristics are consistent with CS requirements for signal sparseness. Therefore, the introduction of CS into the wideband radar imaging system can be expected to reduce data collection quantity, thus to relieve the data storage and processing pressure of the high-resolution radar. Meanwhile, the optimization algorithm can be used to realize the high-resolution imaging through a few pulse echo data.

Besides compressed sensing, the selection of the radar signal directly influences the performance of the distance-Doppler imaging system. The application of the chaotic 
signal for the radar system has many unique advantages, such as random noise characteristic, bandwidth and easy generation [9]. On the other hand, the frequencymodulated signal has high imaging resolution, high output power, low cost, low interception, low interference probability, etc.

\section{Compressed Sensing}

The compressed sensing theory, proposed around 2000, has been rapidly developed in the subsequent several years. According to the compressed sensing theory, if a signal is sparse or is sparse under a certain transformation, then an observation matrix irrelevant to the transformation basis can be adopted to project the high-dimensional signal into the low-dimensional space and afterwards the nonlinear optimization problem can be solved in order to reconstruct the original signal at a high probability through a few observation data. In the theoretical framework, the sampling rate depends on signal structure and content rather than signal width. Additionally, CS theory mainly includes the following three aspects: sparse representation, unrelated sampling and accurate reconstruction of signal [10].

\subsection{Observation Matrix}

The sparse representation of the signal is the precondition for applying the compressed sensing theory. Specifically, a real-valued one-dimensional discrete time signal $x$ with limited length is regarded as $N \times 1$ vector in $R^{N}$ and is recorded as $x[n], n=1,2, \cdots, N$, wherein any signal in $R^{N}$ can be represented by the basis with $\left\{\Psi_{i}\right\}_{i=1}^{N}$ as the column vector. Then, signal $x$ can be expressed as follows:

$$
x=\sum_{i=1}^{N} s_{i} \psi_{i} \text { 或 } x=\Psi s
$$

In the above formula, $s$ is a $N \times 1$ column vector.

We suppose that the description of signal $x$ on basis $\Psi$ is sparse to $S$ ( $\Psi$ is called as sparse matrix) and the condition $S \square N$ can be met, namely: $s_{i}$ in Formula (1) only has $S$ nonzero coefficients. Different from traditional Nyquist sampling theory, the compressed sensing theory aims at non-adaptively and linearly observing the original signal $x$ for $M<N$ times in order to obtain the observed signal ${ }^{y}$ with the length as $M$. Therein, the observation process can be described as follows by a matrix:

$$
y=\Phi x
$$

In the above formula, $\Phi$ is an $M \times N$ observation matrix; Formula (1) is put into the above formula to obtain the following linear observation equation:

$$
y=\Phi x=\Phi \Psi s=\Theta s
$$

In the above formula, $\Theta=\Phi \Psi$ is an $M \times N$ matrix. In many applications, $\Phi$ is a uniform random observation matrix, which does not depend on signal $x$. The schematic diagram of compressed sensing is as shown in Figure1.

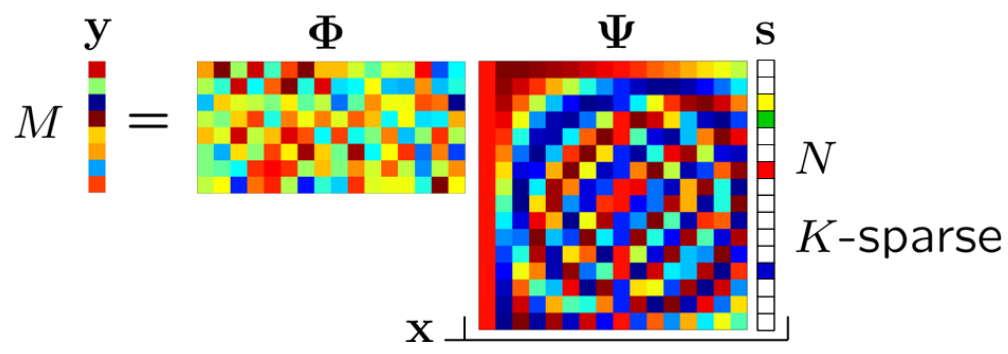

Figure 1. Schematic Diagram of Compressed Sensing 
In the compressed sensing theory, it is very important to design an observation matrix with good performance. Two types of observation matrixes are available: random observation matrix and determined matrix. The observation matrix shall be selected according to the two basic principles: un-correlation and RIP (Restricted Isometry Property). Namely: $\Phi$ in Formula (2) needs to meet RIP principle. For any $S$ sparse signal $x$ and constant $\delta \in(0,1)$, the following condition shall be met [11]:

$$
1-\delta \leq \frac{\|\Phi x\|_{2}^{2}}{\|x\|_{2}^{2}} \leq 1+\delta
$$

If $\Phi$ is fully irrelevant and can meet RIP principle, then $s$ information will be included in $y$. In this way, the original signal can be accurately reconstructed at a high probability. RIP and un-correlation principles can be met at a high probability through selecting the random matrix as the observation matrix.

\subsection{Reconstruction Algorithm}

The reconstruction algorithm aims at adopting the low-dimensional observed signal $y$ to accurately reconstruct the original high-dimensional signal $x$. At present, the reconstruction algorithm is mainly divided into three types: 1) $l_{1}$-norm based convex optimization algorithm; 2) $l_{0}$-norm based greedy algorithm; 3) Combinational algorithm. The convex optimization algorithm is adopted in this article for radar scene reconstruction. Compared with other algorithms, the convex optimization algorithm is based on $l_{1}$-norm for solution and has good reconstruction effect.

\section{Chaotic Imaging Radar based on Compressed Sensing}

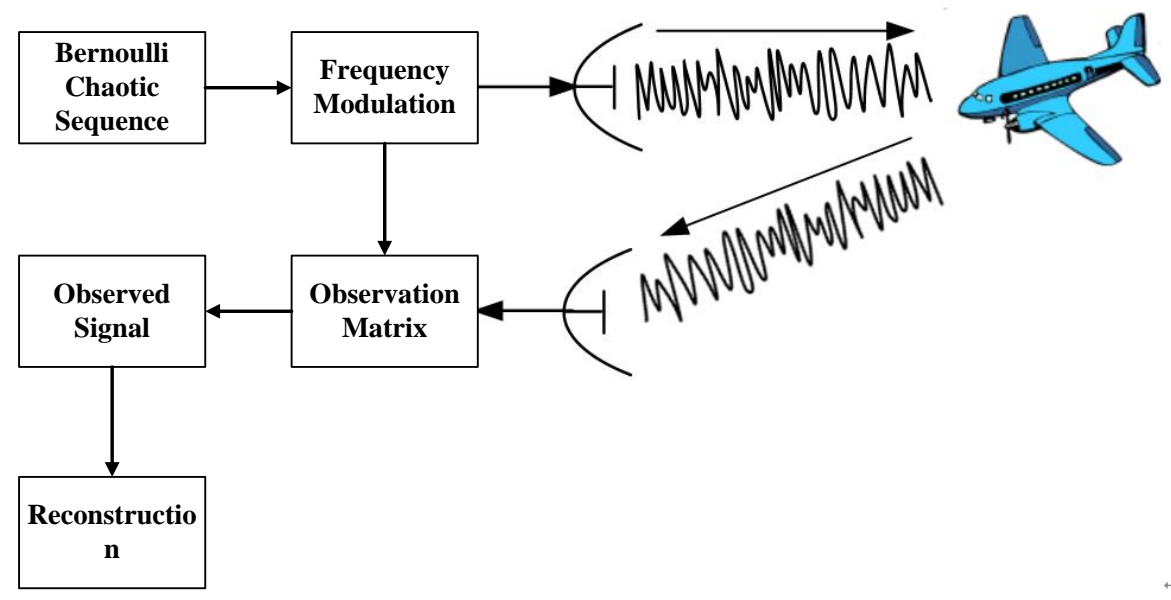

Figure 2. Diagram of Compressed Sensing Chaotic Radar

The compressed sensing chaotic radar system framework is as shown in Figure 2. After frequency modulation, the chaotic sequence is taken as the transmitted radar signal for forming the observation matrix, and the received echo is operated with the observation matrix to obtain the observed signal. Afterwards, the optimization algorithm is adopted to reconstruct the radar scene through the observed data.

The radar echo can be written as the convolution of the transmitted signal and the scene function, namely:

$$
S_{R}(t)=S_{T}(t) * \sigma(t)=\int_{-\infty}^{\infty} S_{T}(t-\tau) \sigma(\tau) d \tau
$$


In the above formula, $S_{R}(t)$ is the radar echo, $S_{T}(t)$ is the transmitted signal and $\sigma(t)$ is the scene scattering function. Through discretization, the above formula can be written into a form of matrix multiplication [12]:

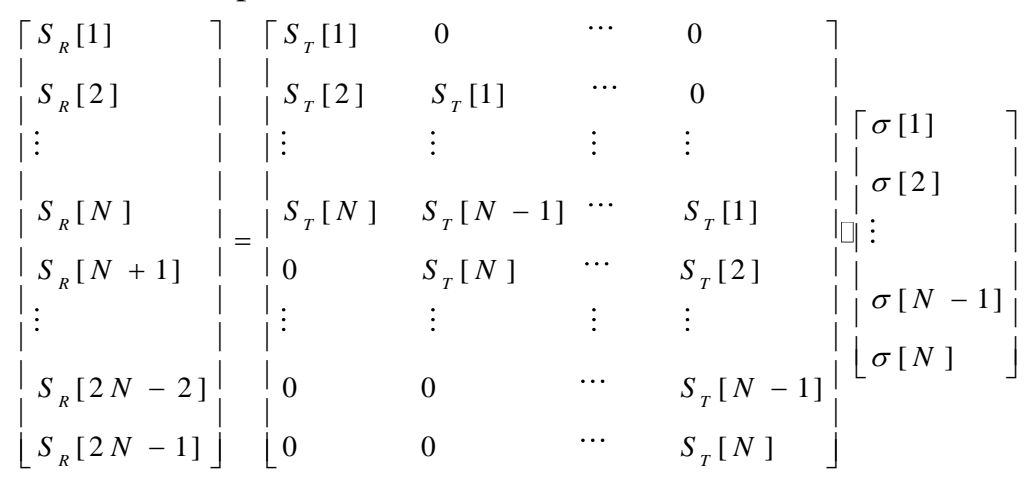

The chaotic signal has random noise characteristic, the autocorrelation function is approximate to $\delta$ function, and the cross-correlation function is approximate to 0. Ever since the 90s of the last century, many scholars have researched the application of chaos in radar signal design and proven the feasibility of the application of chaos in radar signal design. Therein, the research is mainly focused on the following two aspects: chaotic phase-coded signal and chaotic frequency-modulated signal. In the application of the chaotic sequence for frequency modulation, only the sequence generated by Bernoulli mapping can be adopted to obtain ideal autocorrelation characteristic [13]. Therefore, the chaotic sequence generated by Bernoulli mapping is adopted in this article, and Formula (7) is adopted to generate Bernoulli sequence.

$$
\varphi[n+1]=\left\{\begin{array}{l}
B \varphi[n]+1, \varphi[n]<0 \\
B \varphi[n]-1, \varphi[n]>0
\end{array}\right.
$$

Therein, the parameters used for generating the sequence are as follows:

$$
\begin{aligned}
& B=1.7 \\
& \varphi[0] \in[-1 / 2,1 / 2]
\end{aligned}
$$

After Bernoulli sequence is generated, the discrete chaotic function can be obtained according to the following formula:

$$
x[n]=\sum_{k=1}^{n} \varphi[k]
$$

After frequency modulation, the above sequence is taken as the transmitted radar signal $s[n]$. The chaotic frequency-modulated signal is generated by Formula (10):

$$
s[n]=\operatorname{Re}\{A \exp (j 2 \pi K x[n])\}
$$

In the above formula, $A$ is signal amplitude, $K$ is modulation index and $x[n]$ is the discrete chaotic function. The time-domain waveform of the chaotic frequencymodulated signal is as shown in Figure 4, and the autocorrelation function is as shown in Figure 4. 


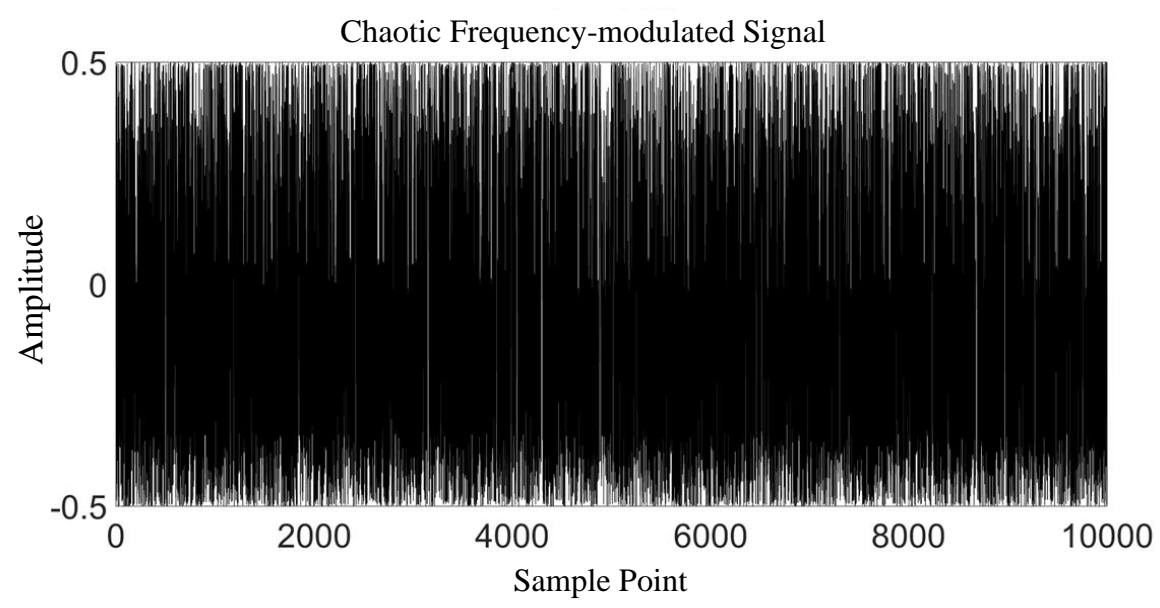

Figure 3. Chaotic Frequency Modulation of Bernoulli

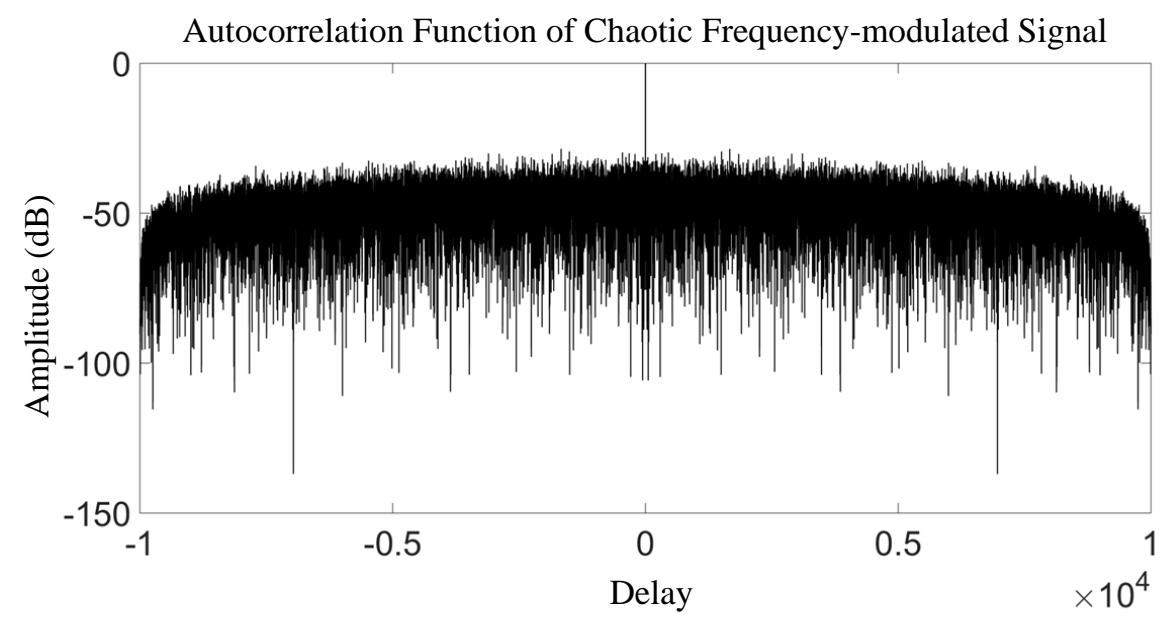

Figure 4. Autocorrelation Function of Chaotic Frequency Modulation

\section{Simulation and Result Analysis}

\subsection{Stationary Target Simulation}

The transmitted radar signal is convoluted with the target to obtain the echo signal. In order to represent the convolution operation by matrix multiplication, the chaotic frequency-modulated signal $s[n]$ is shifted to form the columns of the matrix. Due to the similar noise characteristic of the chaotic frequency-modulated signal, the matrix can be used as the observation matrix in the chaotic radar system based on compressed sensing.

For a stationary target, the target parameters mainly include distance and scattering coefficient. The objects with different scattering coefficients are randomly placed to simulate the radar scene, as shown in Figure 5, wherein the $\mathrm{x}$-axis represents the distance between the target and the radar, and the y-axis represents the scattering coefficient of the target. In order to apply the compressed sensing theory in the simulation, the observed signals shall be less than the sample points of the convolution result, and this can be realized through the down-sampling observation matrix. For the compressed sensing, the columns of the observation matrix need to meet the uncorrelation principle. The correlation of the columns of the observation matrix after down-sampling is as shown in Figure 6. 


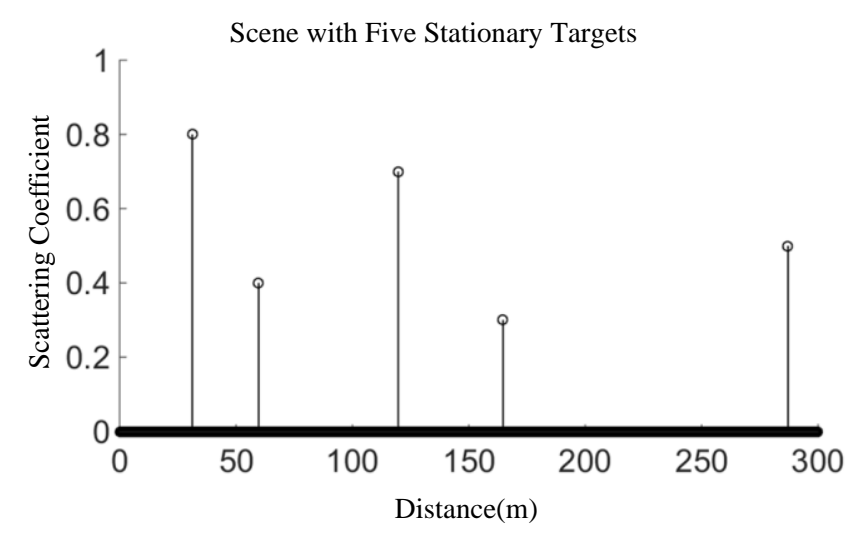

Figure 5. Scene with Five Stationary Targets

Autocorrelation of Columns from Observation Matrix

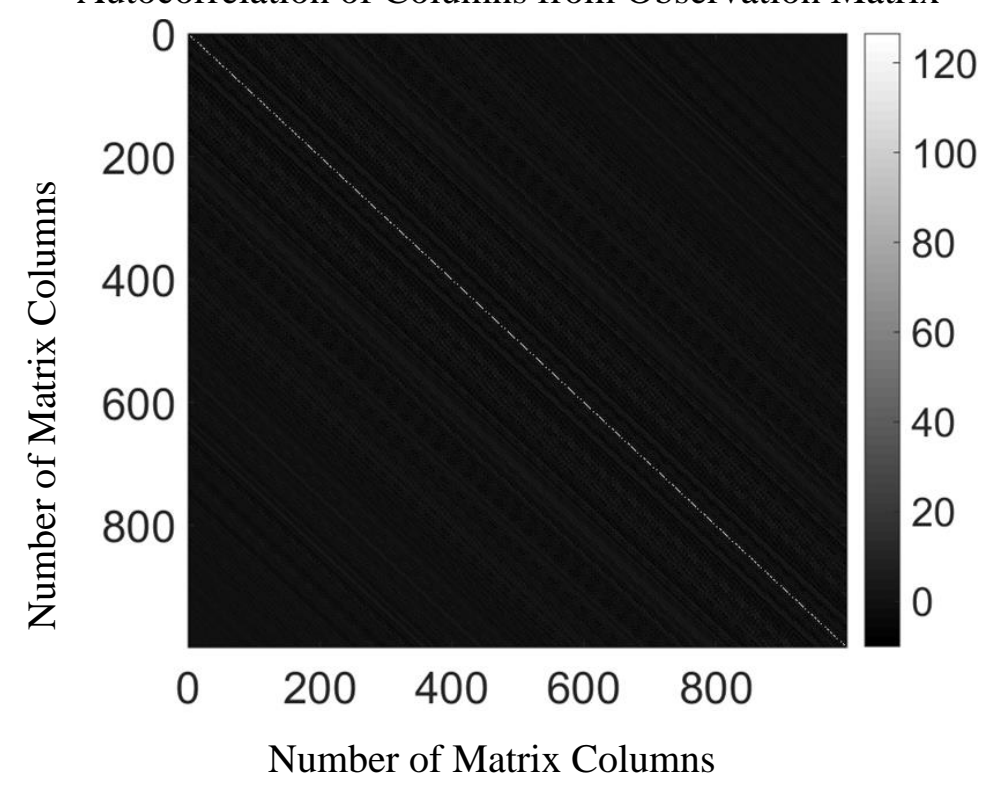

Figure 6. Autocorrelation of Columns from Observation Matrix

For a stationary target, the radar scene is reconstructed through the convex optimization technology mentioned in section 2.2. As shown in Figure 7, the compressed sensing technology can be adopted to accurately reconstruct the radar scene through the under-sampled points. Additionally, the radar scene recovered by the matched filter is as shown in Figure 8. Obviously, many noises are introduced therein during the application of the matched filter for radar scene recovery and enough sample points are required to be obtained in the echo. 


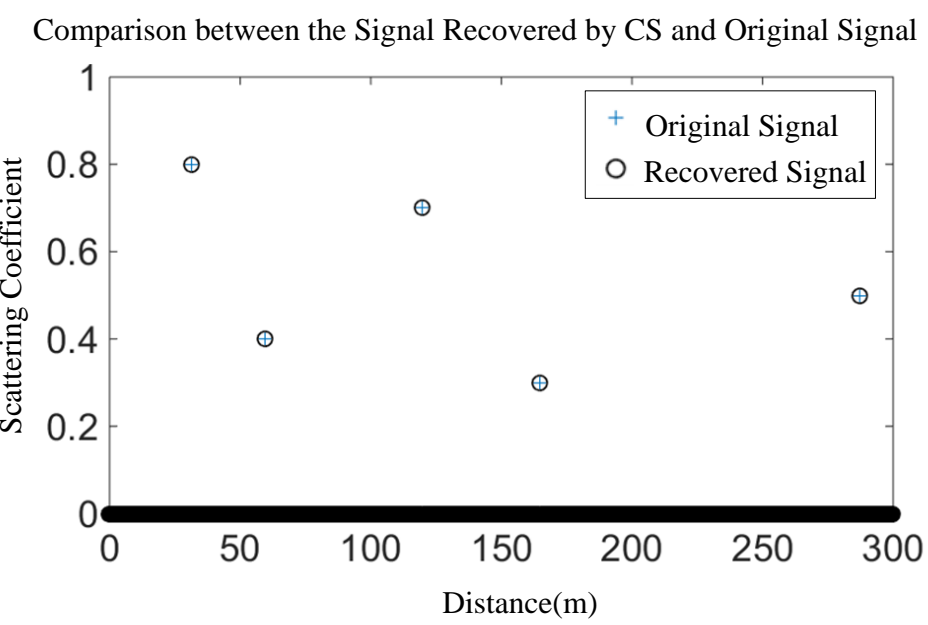

Figure 7. Comparison between the Signal Recovered by CS and Original Signal

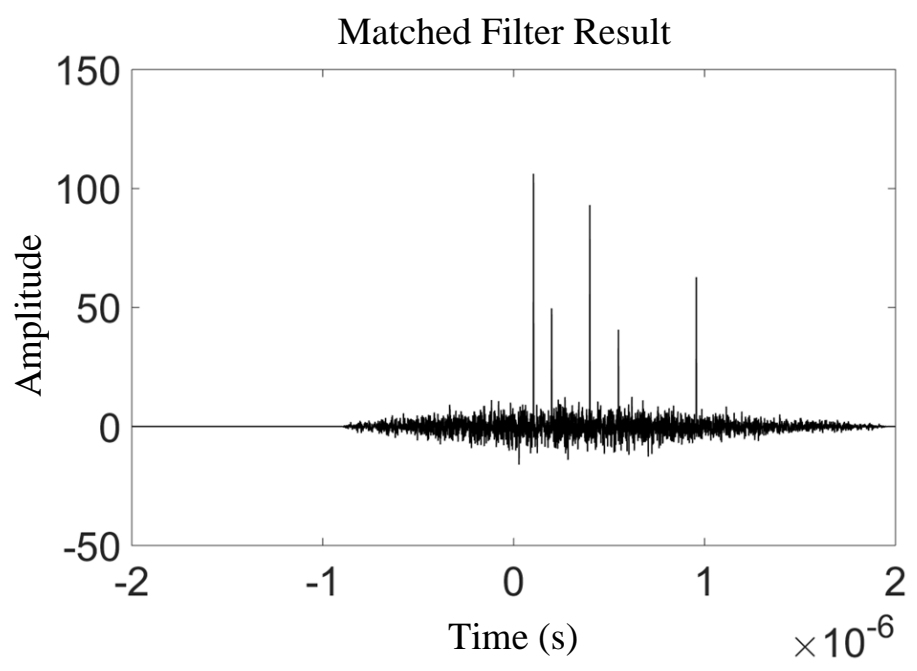

Figure 8. Matched Filter Results

\subsection{Dynamic Target Simulation}

For a dynamic target, Formula (11) is adopted to generate the transmitted signal:

$$
s[n]=\operatorname{Re}\left\{A \exp \left(j 2 \pi K\left(x[n]+f_{d}\right)\right)\right\}
$$

In the above formula, $A$ is signal amplitude, $K$ is modulation index, $x[n]$ is discrete chaotic function, and $f_{d}$ is Doppler frequency. The parameters for generating Bernoulli sequence for the dynamic target are the same as those for the stationary target. As mentioned above, the chaotic frequency-modulated signal generated thereby shall be rearranged to form the observation matrix. Different from the stationary target, for the non-stationary target, all distance signals shall be shifted at each Doppler frequency in order to form the observation matrix, as shown in Figure 9. 


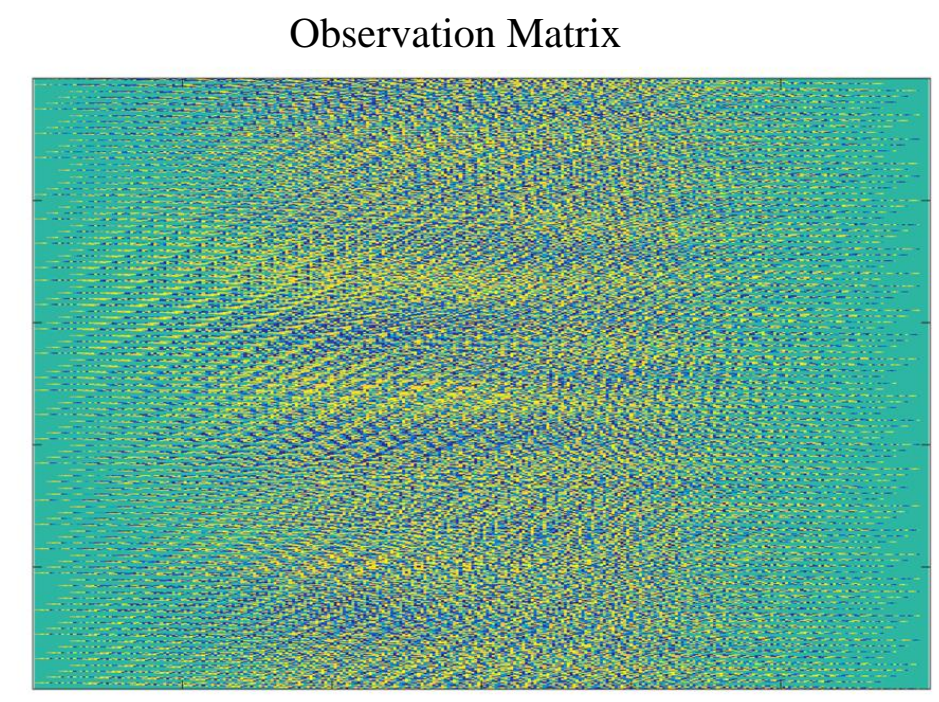

Figure 9. Observation Matrix used for Non-stationary Targets

For a dynamic target, the target parameters mainly include distance and Doppler frequency, and such target can be simulated through the points in a two-dimensional plane, wherein the $\mathrm{x}$-axis represents the distance and the $\mathrm{y}$-axis represents Doppler frequency of the target. The two-dimensional scene generated by the random placement of the targets is as shown in Figure 10. In order to apply the compressed sensing, the scene shall be vectorized into one-dimensional vector. This vector is used to repeat all possible distances for each Doppler frequency. The scene in Figure 10 is vectorized into the vector as shown in Figure 11, namely: the scene including 100 rows and 100 columns in Figure 10 is vectorized into a one-dimensional vector with the length as 10,000 .

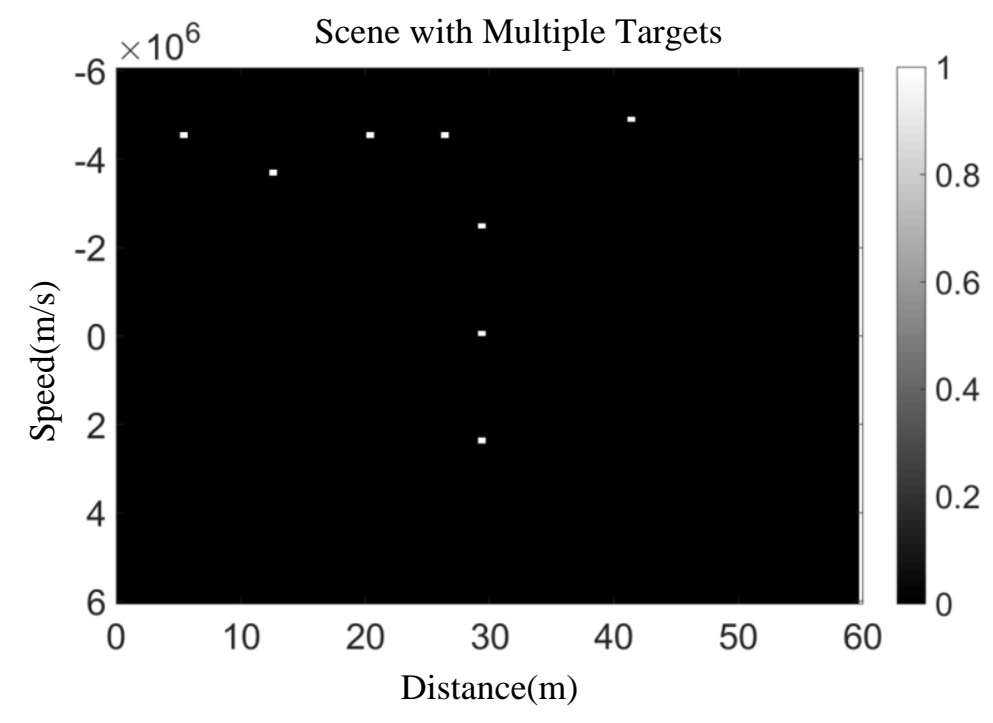

Figure 10. Scene with Multiple Targets 


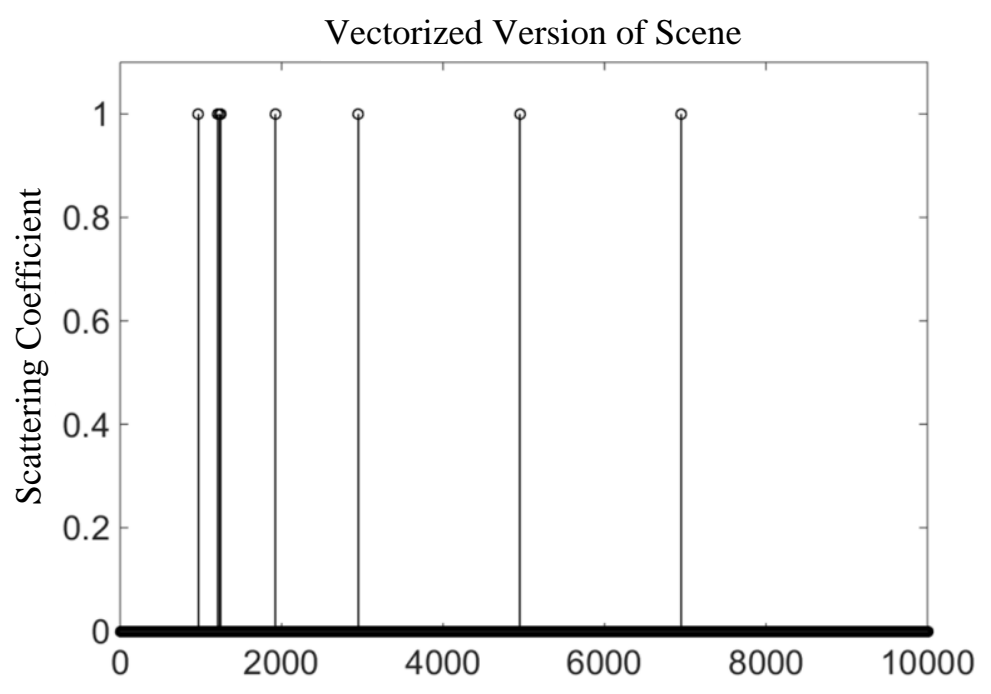

Figure 11. Vectorized Version of Scene

Similarly to the stationary target, in order to apply the compressed sensing, the downsampling operation shall be implemented for the observation matrix and the received signal. At the receiving terminal, the convex optimization technology is adopted for radar scene recovery, as shown in Figure 12. The comparison between the signal recovered by CS and the original signal is as shown in Figure 13, and the radar scene recovered by the matched filter is as shown in Figure 14. Obviously, the matched filter has introduced many noises, and such interference may cover the target or bring difficulty in distinguishing the targets very close to each other.

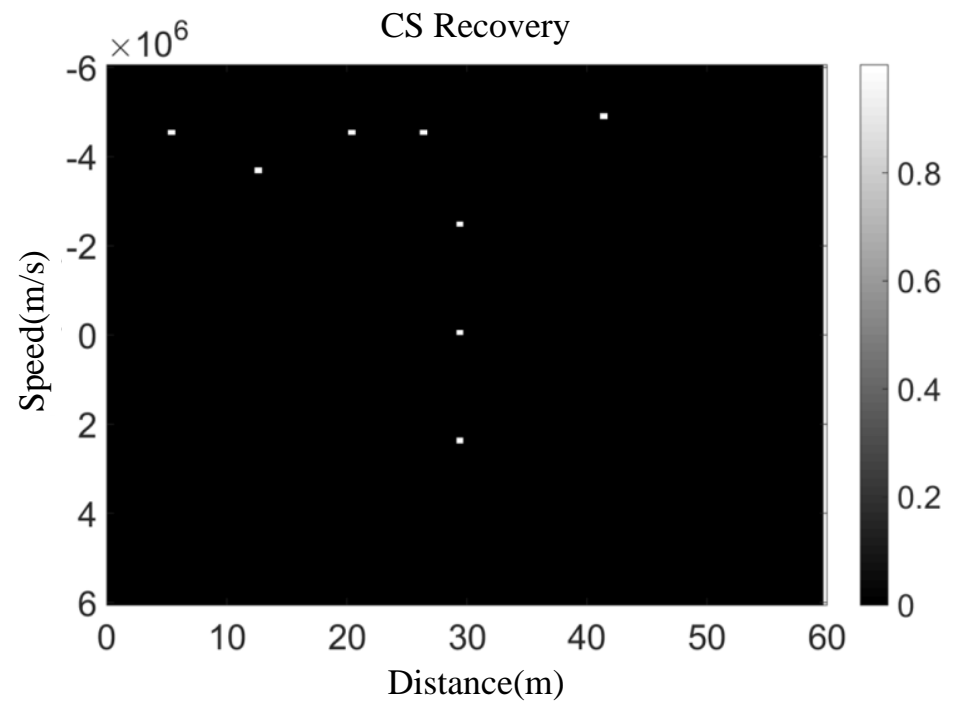

Figure 12. CS Recovered Scene 
Comparison between the Signal Recovered by CS and Original Signal

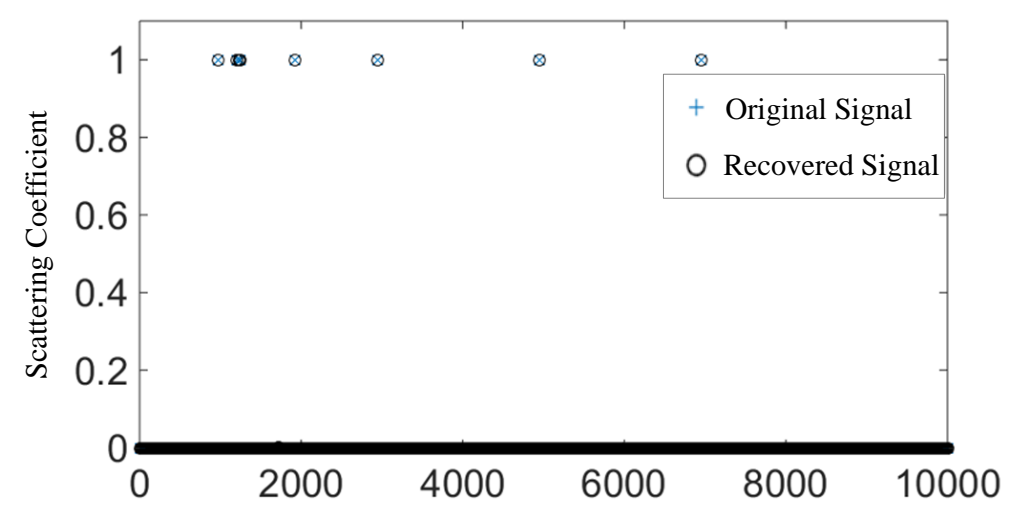

\section{Figure 13. Comparison between the Signal Recovered by CS and Original Signal}

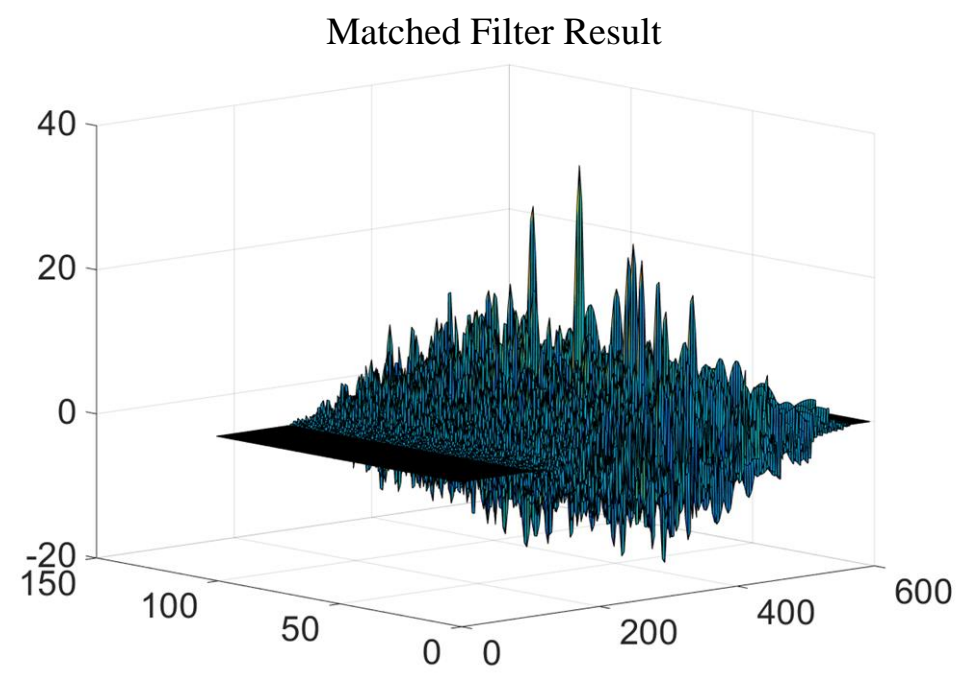

Figure 14. 3-Dimensional Plot of Recovered Scene using Matched Filter

\section{Conclusion}

A compressed sensing method used for improving the resolution of the chaotic radar is proposed in this article. The chaotic sequence generated by Bernoulli mapping is taken as the transmitted signal after frequency modulation, wherein this signal has random noise characteristic, the fuzzy function thereof is approximate to "thumbtack" form and can be directly used for generating the observation matrix. Meanwhile, the stationary and non-stationary target radar scenes are simulated in this article, and the simulation result is also compared with the scene recovered by the matched filter. For a stationary target, the radar scene includes the distance and the scattering coefficient of the target, and the echo signals are down-sampled after passing the observation matrix, and the optimization technology is also adopted to recover the radar scene according to the received signals in the down-sampling process. Compared with the matched filter method, the compressed sensing method has the features of small data volume and good anti-noise performance. For a non-stationary target, the radar scene includes the distance and the speed of the target. In such case, the matched filter can recover the radar scene 
but also introduces many noises at the same time, thus making the target difficult to identify. In comparison, even under the condition of down-sampling the received signals, the compressed sensing method can accurately recover the radar scene. The simulation result shows that the chaotic radar system based on compressed sensing has simple structure and small data processing workload and does not need any matched filter or high-speed analog-digital converter, thus very applicable to the wideband highresolution radar.

\section{References}

[1] X. Song and Y. Geng, "Distributed community detection optimization algorithm for complex networks", Journal of Networks, vol. 9, no. 10, (2014), pp. 2758-2765.

[2] D. Jiang, X. Ying and Y. Han, "Collaborative multi-hop routing in cognitive wireless networks", Wireless Personal Communications, (2015), pp. 1-23.

[3] Z. Lv, A. Halawani and S. Feng, "Multimodal hand and foot gesture interaction for handheld devices", ACM Transactions on Multimedia Computing, Communications, and Applications (TOMM), vol. 11, no. 11s, (2014), p. 10.

[4] G. Liu, Y. Geng and K. Pahlavan, "Effects of calibration RFID tags on performance of inertial navigation in indoor environment", 2015 International Conference on Computing, Networking and Communications (ICNC), (2015).

[5] J. He, Y. Geng, Y. Wan, S. Li and K. Pahlavan, "A cyber physical test-bed for virtualization of RF access environment for body sensor network, IEEE Sensor Journal, vol. 13, no. 10, (2013), pp. 38263836.

[6] W. Huang and Y. Geng, "Identification Method of Attack Path Based on Immune Intrusion Detection", Journal of Networks, vol. 9, no. 4, (2014), pp. 964-971.

[7] X. Li, Z. Lv and J. Hu, "XEarth: A 3D GIS Platform for managing massive city information", Computational Intelligence and Virtual Environments for Measurement Systems and Applications (CIVEMSA), 2015 IEEE International Conference on IEEE, (2015), pp. 1-6.

[8] J. He, Y. Geng, F. Liu and C. Xu, "CC-KF: Enhanced TOA Performance in Multipath and NLOS Indoor Extreme Environment", IEEE Sensor Journal, vol. 14, no. 11, (2014), pp. 3766-3774.

[9] N. Lu, C. Lu, Z. Yang and Y. Geng, "Modeling Framework for Mining Lifecycle Management", Journal of Networks, vol. 9, no. 3, (2014), pp. 719-725.

[10] Y. Geng and K. Pahlavan, "On the accuracy of rf and image processing based hybrid localization for wireless capsule endoscopy”, IEEE Wireless Communications and Networking Conference (WCNC), (2015).

[11] X. Li, Z. Lv and J. Hu, "Traffic management and forecasting system based on 3d gis", Cluster, Cloud and Grid Computing (CCGrid), 2015 15th IEEE/ACM International Symposium, (2015), pp. 991-998.

[12] S. Zhang and H. Jing, "Fast log-Gabor-based nonlocal means image denoising methods", Image Processing (ICIP), 2014 IEEE International Conference on IEEE, (2014), pp. 2724-2728.

[13] D. Jiang, Z. Xu and Z. Chen, "Joint time-frequency sparse estimation of large-scale network traffic", Computer Networks, vol. 55, no. 15, (2011), pp. 3533-3547.

[14] J. Hu, Z. Gao and W. Pan, "Multiangle Social Network Recommendation Algorithms and Similarity Network Evaluation", Journal of Applied Mathematics, (2013).

[15] J. Hu and Z. Gao, "Modules identification in gene positive networks of hepatocellular carcinoma using Pearson agglomerative method and Pearson cohesion coupling modularity", Journal of Applied Mathematics, (2012).

[16] Z. Lv, A. Tek and F. D. Silva, "Game on, science-how video game technology may help biologists tackle visualization challenges", PloS one, vol. 8, no. 3, (2013), p. 57990.

[17] T. Su, W. Wang and Z. Lv, "Rapid Delaunay triangulation for randomly distributed point cloud data using adaptive Hilbert curve", Computers \& Graphics, vol. 54, (2016), pp. 65-74.

[18] S. Zhou, L. Mi, H. Chen and Y. Geng, "Building detection in Digital surface model", 2013 IEEE International Conference on Imaging Systems and Techniques (IST), (2012).

[19] J. He, Y. Geng and K. Pahlavan, "Toward Accurate Human Tracking: Modeling Time-of-Arrival for Wireless Wearable Sensors in Multipath Environment”, IEEE Sensor Journal, vol. 14, no. 11, (2014), pp. 3996-4006.

[20] Z. Lv, A. Halawani and S. Fen, "Touch-less Interactive Augmented Reality Game on Vision Based Wearable Device", Personal and Ubiquitous Computing, vol. 19, no. 3, (2015), pp. 551-567.

[21] G. Bao, L. Mi, Y. Geng, M. Zhou and K. Pahlavan, "A video-based speed estimation technique for localizing the wireless capsule endoscope inside gastrointestinal tract", 2014 36th Annual International Conference of the IEEE Engineering in Medicine and Biology Society (EMBC), (2014).

[22] D. Zeng and Y. Geng, "Content distribution mechanism in mobile P2P network", Journal of Networks, vol. 9, no. 5, (2014), pp. 1229-1236. 
[23] W. Gu, Z. Lv and M. Hao, "Change detection method for remote sensing images based on an improved Markov random field", Multimedia Tools and Applications, (2015), pp. 1-16.

[24] Z. Chen, W. Huang and Z. Lv, "Towards a face recognition method based on uncorrelated discriminant sparse preserving projection", Multimedia Tools and Applications, (2015), pp. 1-15.

\section{Author}

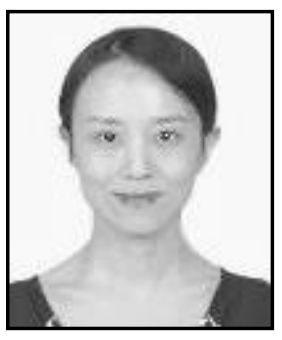

Li Wenjuan, she received her M.S. degree in Communication and Information System from Liaoning Technical University in Liaoning, China. She is currently a lecturer in the College of Mobile Telecommunications Chongqing University of Posts and Telecom. Her research interest is mainly in the area of Mobile Communication, Signals and Systems. She has published several research papers in scholarly journals in the above research areas and has participated in several books. 